\title{
Comparison between Long and Transverse Lung Ultrasound in Critically Ill Patient
}

Y.E. Rezk ${ }^{1}$, M.A.Algazzar ${ }^{1}$, A.M. Almenshawy ${ }^{2}$ and O.S.Mahana ${ }^{3}$

${ }^{1}$ Cardio Thoracic Dept., Faculty of Medicine, Benha Univ., Benha, Egypt

${ }^{2}$ Critical Cares Dept., Faculty of Medicine, Alexandria Univ., Alexandria, Egypt

${ }^{3}$ Critical Care Dept, Qabbary General Hospital, Egypt

E-Mail:drosama.mahana@yahoo.com

\begin{abstract}
Lung ultrasound used for semi-quantification of lung aeration and assessment of lung pathology. lung scan in the longitudinal approach could be identified in between the ribs. Transverse scan has the ability of visualization of longer pleural and more artifacts using lung scoring system or pathological identification. We included 50 patients from Benha University Hospital and Alexandria main university hospital eligible for our study scanned by lung ultrasound in long and transverse scanning technique and do comparison using CT scan as a gold standard. The study proved that transverse technique show more artifact and had both sensitivity and specificity for all lung pathology included.
\end{abstract}

Keywords: Lung ultrasound, Transverse technique, Long technique.

\section{Introduction}

Lung ultrasound has become a fast, easy accessible, bedside essential diagnostic and therapeutic tool in critically ill patient for rapidly identifying the etiology, following treatment progress, guiding invasive procedures, clarifying nonspecific chest radiograph (CXR) abnormalities and when used in combination with cardiac and vascular ultrasound, it can enhance the understanding of etiology and may reduce the need for CXR or chest Computerized Tomography (CT) $[1,2]$.

Chest ultrasound has better diagnostic accuracy and sensitivity than portable chest radiography in diagnosing common lung pathologies as pneumothorax, pleural effusion, Acute Interstitial Syndrome (AIS), and consolidation. In addition, lung ultrasound can be performed bedside and can be repeated to follow progress. It is valuable alternative to CT chest $[3,4]$.

Lung ultrasound is a complex procedure; each target area is visualized in real time by multiple crosssectional images in several planes. Lungs and pleura are best evaluated by modified application of the transducer in the transverse and oblique intercostal positions as ribs may interfere with lung, and pleura visualization $[5,6]$.

Lung ultrasound allows semi-quantification of lung aeration and assessment of lung pathology based on number/coalescence of vertical artifacts (B-lines) in the longitudinal lung scan and the pleura is identified between two ribs. Transverse scan has shown to visualize longer pleural and more artifacts using lung score. However, the sensitivity of either the longitudinal or the transverse scan in detecting common lung pathologies has not been identified [7].

Pleural effusion: Anechoic zone separating the parietal and visceral pleura with change of shape as a function of respiration. Abolished lung sliding and the stratosphere sign: the absence of lung sliding is a basic first step for the diagnosis of pneumothorax. The abolition of lung sliding generates the stratosphere sign on M-mode. The B-lines (interstitial lung syndromes): it is a vertical narrow-based artifact that spreads out to the edge of the screen. The tissue-like sign (lung consolidation): the tissue-like sign indicates an image is echogenic like a liver, and which behaves like a tissue, that is, does not generate any sinusoidal sign. The Alines (normal lung surface): this is a reverberation artifact of the pleural line located deeper at the same distance as the skin-pleural line distance[8,9].

\section{Patients and methods}

What A total of 50 patients were enrolled consequentially in the present study. They were selected from those admitted to Benha University Hospital and Alexandria University Hospitals with respiratory complains on admission.

Informed consent will be taken from next of kin for every patient before enrollment in the study. The study were approved from the local ethics committee of the Faculty of Medicine - Benha University.

\section{Eligibility}

Both genders are eligible for the study.

Inclusion Criteria:

- Adult ( $\geq 18$ years old).

- Patients with acute respiratory complains including dyspnea, respiratory distress, cough, expectoration or cyanosis.

- Patient with respiratory signs including paradoxical breathing, hypoxia, crackles, wheezes, hypercapnia

\section{Exclusion Criteria}

- Patients admitted with definite diagnosis of respiratory complain.

- Patient less than 18 years

- Patient with chest wall deformity or abnormality interfere with chest ultrasound examination as obesity( $\mathrm{BMI}<30$ )

\section{Methods}

Study design

Observational cohort study .

\section{Participants}

The study will be conducted on 50 patients with acute respiratory complain who will be admitted to Benha University Hospital and Alexandria University Hospital after being assessed for eligibility for the 
study. This will be done by taking detailed history from the patient's relatives and through clinical examination.

\section{A. Stabilization and assessment}

All patients enrolled in this study diagnosed as acute respiratory complains were subjected to the following:

1- Initial assessment (ABCs) as regard:

a) Airway patency and security.

b) Breathing assessment and oxygen supply.

c) Circulation maintenance via intravenous fluids or vasopressors.

2- Demographic data including age (years) \& sex.

3- Complete medical history including (diabetes mellitus-hypertension -smoking).

4- Complete physical examination including \{respiratory rate -heart rate -temperature -mean blood pressure - spo $2-\mathrm{ABG}$ )

5- Chest ultrasound examination using portable ultrasound system equipped with a P21 (1-5 MHz) phased-array transducer as follows;

- Longitudinal and transverse scans will be performed in six areas of each lung (anterior, lateral and posterior) each divided to superior and inferior.

- Once longitudinal scan will be performed, transverse scan will be obtained by a probe rotation until the ribs disappeared.

- Physicians performing the lung ultrasound examinations were not involved in the patient's care and were blinded to history, laboratory data, imaging, treatments, and diagnosis.

6- CT chest without contrast to delineate lung pathology.

\section{B. Measurements}

The following data will be collected from all patients;

1- Demographic data of the patient (age and gender), primary diagnosis, need of mechanical ventilation

2- Comparison between the findings noted in both transverse and longitudinal scans to detect lung pathology.

3- Diagnosis of lung pathology as shown by CT Chest

4- All data will be collected and analyzed statistically.

\section{Results}

A total part have been examined 600 area the negative parts which show normal lung sliding and Aprofile in long ultrasound was 250 which was $41.66 \%$ of total parts examined when transverse show 205 parts which was $34.16 \%$ of total parts examined and negative parts in CT chest was 192 part which was $32 \%$ of parts of comparison as illustrated in Table (2), Fig (1).
Table (1) Demographic data.

\begin{tabular}{lc}
\hline Age & $>18$ yrs \\
\hline Males & 28 \\
Females & 22 \\
Co morbidities & \\
Diabetes & $17 \%$ \\
Hypertension & $18 \%$ \\
IHD & $26 \%$ \\
COPD & $21 \%$ \\
\hline
\end{tabular}

Table (2) Negative parts shown in examination.

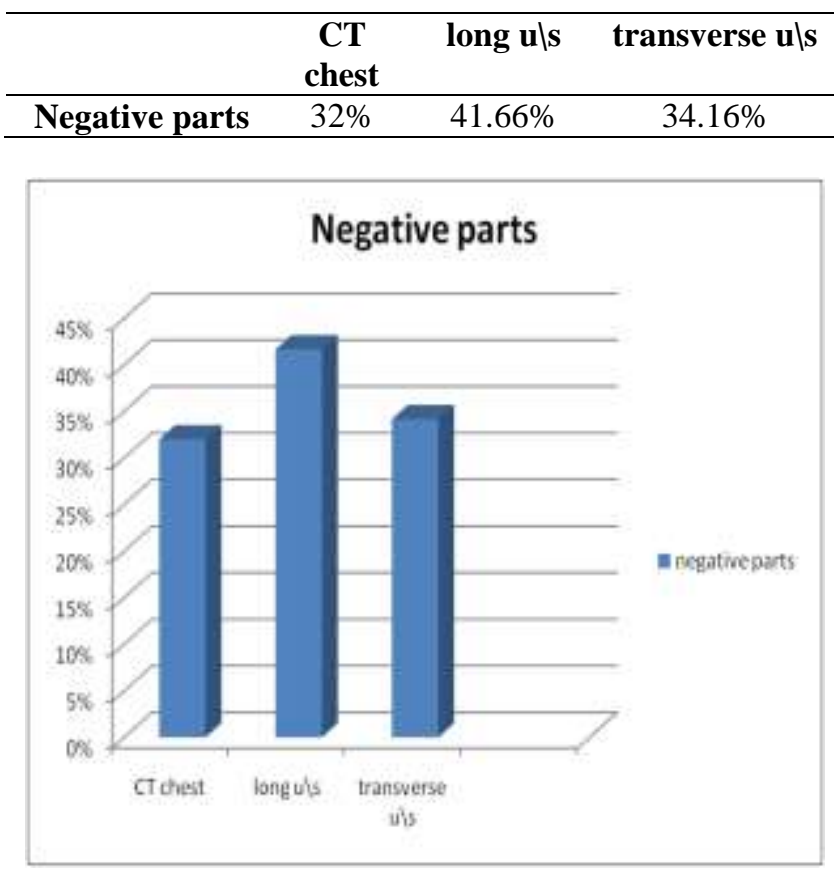

Fig (1) Negative parts shown in examination.

And regarding the parts or areas of examination which show positive finding using $\mathrm{CT}$ chest as gold standard which 408 area show pathological finding we $f$ that long lung uls 350 area could show positive finding which represent sensitivity $85.78 \%$ and 323 area show findings with diagnosis was the same as reported in CT chest which represent $79 \% .16 \%$ specificity on the other hand transverse lung uls the number of 395 area of examination show positive finding which represent $96.81 \%$ sensitivity and 375 of these areas show diagnosis was the same as reported as CT chest which represent $91.91 \%$ specificity as summated and illustrated in Table (3), Fig (2).

Table (3) Total sensitivity and specificity of positive parts examined.

\begin{tabular}{lcc}
\hline & long uls & transverse uls \\
\hline sensitivity & $85.78 \%$ & $97 \%$ \\
specificity & $79.16 \%$ & $91.90 \%$ \\
\hline
\end{tabular}




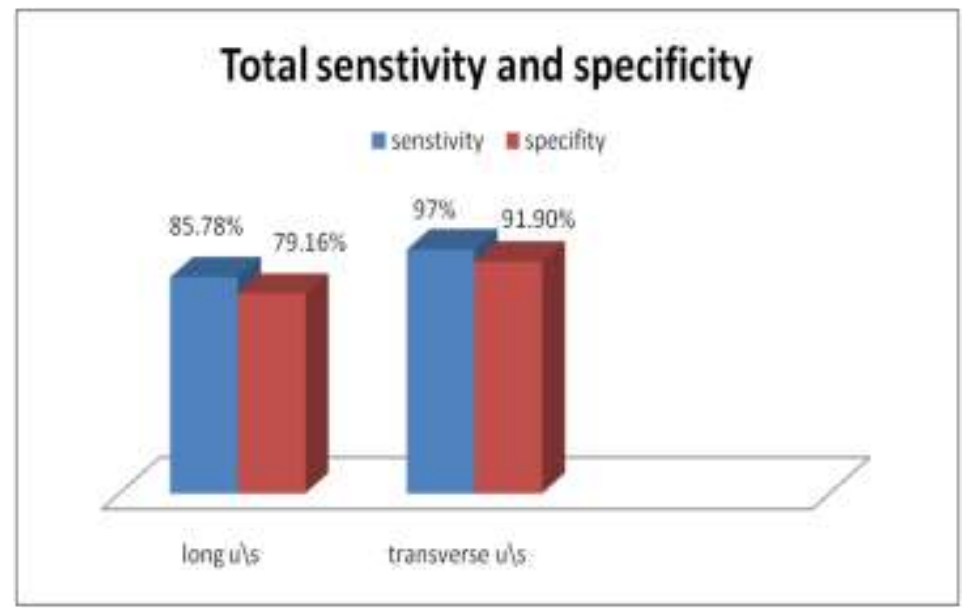

Fig (2) Total sensitivity and specificity of positive parts examined.

The As regard pathological diagnosis the sensitivity and specificity could be compared between two techniques as follow: Table $(4,5)$, Fig $(3,4)$.

1)Congestion diagnosed in 150 area on CT chest when 138 artifact shown in long uls (sensitivity 92\%) and 135 of these artifacts diagnosed by uls as congestion (specificity 90\%) when 148 artifact could be detected in these areas by transverse uls ( sensitivity 98.66\%) and 142 of these artifacts diagnosed as congestion by transverse uls (specificity 94.67\%).

2)Consolidation due to different causes detected in 145 areas in CT chest, 113 artifact detected in these areas by long uls (sensitivity $77.93 \%$ ) and 94 of these artifacts diagnosed consolidation by long uls (specificity $64.82 \%$ ) when 140 artifact detected in these areas by transverse uls (sensitivity $96.55 \%$ ), 130 of these artifacts diagnosed as consolidation by transverse uls (specificity $89.65 \%$ )

3)Effusion detected in 47 area in CT chest, 41 artifact could be detected in these areas by long uls (sensitivity $87.23 \%$ ) ,39 of these artifacts diagnosed as effusion by long uls (specificity $82.97 \%$ ), when 45 artifact detected on these areas by transverse uls (sensitivity $95.7 \%$ ) and 43 of these artifacts diagnosed as effusion by transverse uls (specificity $91.48 \%$ )

4)Effusion with atelectasis Iconsolidation detected in 28 area on CT chest, 24 artifact detected on these areas by long uls (sensitivity $85.71 \%$ ) and 23 of these artifacts diagnosed effusion with underlying atelectasis Iconsolidation by long uls (specificity $82.21 \%$ ) when 26 artifact detected in these areas by transverse uls (sensitivity $92.89 \%$ ) and 25 of these artifacts diagnosed as effusion with underlying atelectasis Iconsolidation by transverse uls (specificity $89.2 \%$ )

5)pneumothorax detected in 38 area in CT chest, 34 artifact could be detected in these areas by long uls (sensitivity $89.47 \%$ ), 32 of these artifacts diagnosed as effusion by long uls (specificity $84.21 \%$ ), when 36 artifact detected on these areas by transverse uls (sensitivity 94.73\%) and 35of these artifacts diagnosed as effusion by transverse uls (specificity $92 \%$ ).

Table (4) Comparison of sensitivity between long and transverse LUS.

\begin{tabular}{lccccc}
\hline & Congestion & Consolidation & Effusion & $\begin{array}{c}\text { Effusion with } \\
\text { atelectasis }>\text { consolidation }\end{array}$ & pneumothorax \\
\hline Long uls & $92 \%$ & $77.93 \%$ & $87.23 \%$ & $85.71 \%$ & $89.47 \%$ \\
transverse uls & $99 \%$ & $96.55 \%$ & $95.70 \%$ & $92.85 \%$ & $94.73 \%$ \\
\hline
\end{tabular}

Table (5) comparison of specificity between long and transverse LUS.

\begin{tabular}{lccccc}
\hline & Congestion & Consolidation & Effusion & $\begin{array}{c}\text { Effusion with atelectasis } \\
\text { lconsolidation }\end{array}$ & pneumothorax \\
\hline Long uls & $90 \%$ & $64.82 \%$ & $82.97 \%$ & $82.14 \%$ & $84.21 \%$ \\
Transverse uls & $94.67 \%$ & $89.65 \%$ & $91.48 \%$ & $89.20 \%$ & $92.10 \%$ \\
\hline
\end{tabular}




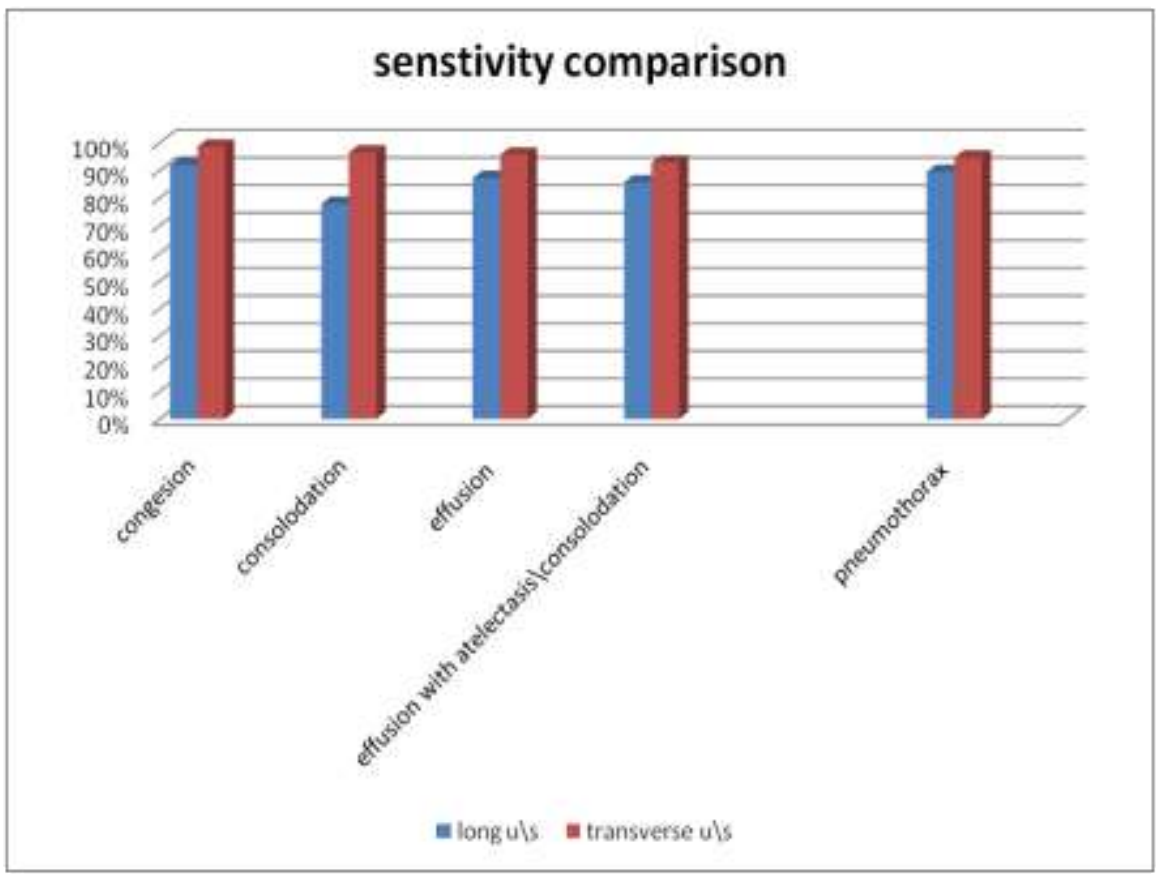

Fig (3) Comparison of sensitivity between long and transverse LUS

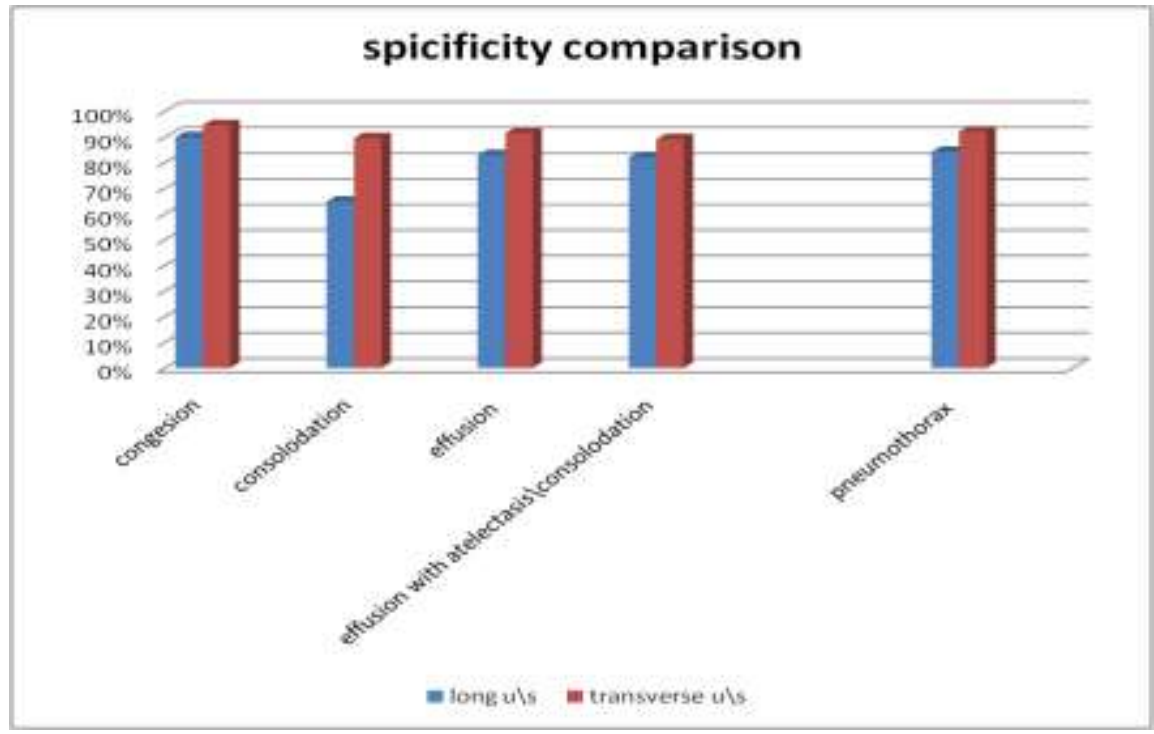

Fig (4) comparison of specificity between long and transverse LUS

\section{Discussion}

A few investigations completed on distinguish the vitality of ultrasonography midsection for clinical act particularly to emergency unit units whichever by and large or to particular pathology alternately for evaluation for particular systems or conventions of the examination.

Ribs meddle with visualization of lung tissue, pathologies What's more by modifying those probe position should be obvious additional artifacts, What's more finer appraisal for lung air circulation.
As stated by investigation [3] selected 38 tolerant for ultrasonography examining by the long What's more transverse strategy for downright part analyzed 456 parts in length ultrasonography indicate 46. $2 \%$ negative examination (A-profile) when transverse show 34. $2 \%$ negative examination. (10). To our examine the long ultrasonography demonstrate $41.66 \%$ about downright parts indicate negative examination At transverse hint at $34.16 \%$ from claiming downright parts inspected demonstrate negative examination Anyhow to our examine we utilized ct midsection Concerning illustration An gold standard which 
demonstrate $32 \%$ of aggregate regions with negative report, Furthermore there may be $2.16 \%$ false negative bring about transverse strategy What's more 9. $66 \%$ false negative bring about long system.

There may be assention clinched alongside two investigations that in length procedure demonstrate All the more negative examination over transverse which substantiate that transverse strategy show a greater amount relic Anyway in our ponder utilizing ct midsection as gold standard settle on us recognize that also transverse LUS hint at false negative effects (2. $16 \%$ ) which Might not a chance to be turned out by [3] consider, which make our investigation All the more exact [10].

In regards will certain brings about [3] utilized a scoring framework as stated by vicinity for B-lines , arrange the blockage with gentle Also direct, a third classification hold extreme blockage, consolidation, radiation, atelectasis as opposed our contemplate a spot analysis recognized Toward lung ultrasonography done both systems, this is more useful over [3] consider. (10). Likewise over our contemplate we analyze both systems with ct midsection Concerning illustration An gold standard along these lines we might evaluate the affectability What's more specificity same time over [3] the certain discovering contrasted with the aggregate parts inspected also others affectability Also specificity Might not be assessed. (10). As stated by in turn investigation [11] those ponder which point to assessment those affectability Also specificity of LUS in examination with CXR, they likewise utilized ct midsection Concerning illustration a gold standard with inconclusive system favoring, Previously, their ponder also those examiners assess the patients clinically What's more for research center examination When tolerant examination Eventually Tom's perusing lung ultrasonography as opposed our contemplate [11] .

Consider bring about shortages indicate affectability should pneumonia $94 \%$, specificity $93 \%$ same time our contemplate demonstrate affectability to combination in the least affectability clinched alongside in length us $77 \%$, transverse $96.55 \%$ specificity On in length us $64.82 \%$, transverse us $89.65 \%$ [11]. Viewing blockage alternately edema [11] study hint at affectability $93 \%$,specificity $91 \%$ same time our investigation indicate long us affectability $92 \%$ ,specificity $90 \%$,, transverse us affectability $98.66 \%$, specificity $94.67 \%$. (11). In regards to radiation [11] consider hint at affectability $94 \%$ specificity $96 \%$, our study indicate in length us affectability $87.23 \%$ specificity 82.975 , transverse us affectability $95.7 \%$ ,specificity 91. 48\%. Viewing pneumothorax [11], demonstrate affectability $100 \%$, specificity $98 \%$ same time our consider show in length us affectability 89. $47 \%$,specificity $84.21 \%$, transverse us affectability 94. $73 \%$ specificity 92. 1\%. [11]. An incredible Contrast clinched alongside affectability Also affectability the middle of [11] Also long us strategy Furthermore it Might make clarified grade because of rib impedance Similarly as in [11] they not notice any procedure, they might would both Also think about those best result [11]. Also different contrasts Might make distinguished between our consider Furthermore [11] for example, such that pneumonia we bring About the same affectability Anyway low specificity , in clogging we bring higher affectability Furthermore specificity for transverse strategy, almost the same affectability Furthermore specificity done long us ,, in regards to radiation checked Contrast Previously, in length us ,, same affectability done transverse system , low specificity, in regards pneumothorax we need low affectability Also specificity which stamped Previously, long, lesquerella stamped to transverse [11]. This contrasts Might make clarified because of ,, over [11] 0the analyst turned by clinical examination, research center investigations which regulate him on spot finding Toward ultrasonography make specificity Contrast , Additionally for their consequence At whatever tolerant for finding acknowledged as certain done outcome same time On our consider we ascertain the effect as stated by every part needed been inspected something like that Previously, our investigation same tolerant might indicate sure Also negative as stated by parts analyzed Also consequence this bringing down the both affectability What's more specificity for example, such that pneumothorax, over [11] if particular case piece inspected in the tolerant hint at standard code sign those tolerant will setting off should sure bring about difference with our consider those just inspected parts whish hint at certain will be acknowledged and the negative no acknowledged [11]. An additional reason for this distinction is because of that On our ponder we place another class hint at those radiation for superadded merging with or without atelectasis On [11] this classification not embedded along these lines whatever of single finding things Might a chance to be included or evacuated in this examine Similarly as they must compose a solitary analysis which might influence the conclusion outcome What's more make An distinction yet all the to outline judgment both investigations demonstrate the vitality about lung ultrasonography Also our examine good particular case method [11]. Their ponder demonstrate upper hand for relationship between clinical examination Furthermore finding as well as it might influence the effect same time our consider demonstrate All the more precision which might make recognized because of count those aggregate bring about shortages as stated by aggregate parts need been examined, expansion of more classifications for finding and finally those analyst might have been veiled regarding clinical What's more research center finding Furthermore make our finding purely from radiological perspective of perspective which aggravate us not guided Toward clinical situation.

Concerning illustration in regards to [12] similar contemplate between at midsection radiograph clinched alongside critically sick tolerant. In this investigation they incorporate 42 patient, know patients were mechanically ventilated, no other avoidance criteria. 
[12]. They sorted those patients with four pathologic categories, same technique, not think about in length alternately transverse, they Think as of those best perspective Furthermore driver might have been blind regarding tolerant data, diagnosis, other radiological investigation.

At we think about those four obsessive classifications for [12] comes about we discover that pneumothorax toward [12] examine show affectability $75 \%$, specificity $93 \%$ The point when over our investigation demonstrate in length us affectability 89 . $47 \%$,specificity $84.21 \%$, transverse us affectability 94. $73 \%$ specificity 92. 1\%. [12] Likewise viewing affectability show low effect starting with both in length and transverse ultrasonography which Might make demonstrated because of no Choice criteria which make a greater amount masker of the certain finding, Similarly as view specificity [12] study demonstrate All the more specificity over in length ultrasonography Might make demonstrated Eventually Tom's perusing masker of the ribs Anyway demonstrate almost same brings about transverse ultrasonography [12]. Likewise view merging [12] investigation hint at affectability $100 \%$, specificity $78 \%$ The point when over our examine indicate affectability should merging whatsoever affectability for in length us $77 \%$, transverse $96.55 \%$ specificity to long us $64.82 \%$, transverse us $89.65 \%$. [12]. demonstrate helter skelter affectability over both in length What's more transverse ultrasonography clarified Concerning illustration they not recognize particular technobabble and they utilize the best finding and the that's only the tip of the iceberg Contrast from long demonstrated Eventually Tom's perusing ribs shadow. Also Similarly as viewing specificity [12] examine demonstrate secondary affectability over in length ultrasonography Furthermore low over transverse, this Might a chance to be demonstrated Toward also ribs shadow masker those combination over in length ultrasound, Furthermore transverse ultrasonography demonstrate that's only the tip of the iceberg specificity Concerning illustration On our investigation we incorporate every one merging not pneumonia best. (12). Viewing radiation [12] examine indicate affectability Also specificity of $100 \%$ The point when over our ponder demonstrate long us affectability $87.23 \%$ specificity 82.975 , transverse us affectability $95.7 \%$,specificity $91.48 \%$.

There may be incredible Contrast the middle of [12] consider What's more in length ultrasonography to both affectability What's more specificity Might a chance to be demonstrated by ribs shadow Furthermore About with transverse ultrasonography with gentle distinction Might a chance to be demonstrated Concerning illustration we figure the outcomes as stated by tolerant inspected not the patients number. (12). Likewise view blockage [12] examine aftereffect affectability $94 \%$,specificity $93 \%$ Also On our consider hint at long us affectability $92 \%$,specificity $90 \%$, transverse us affectability 98. 66\%,, specificity 94. $67 \%$ which almost those same effects. (54). Those net
Contrast between [12] Furthermore our ponder because of system for calculation, they best select mechanically ventilated patients, no avoidance criteria Also we utilized two systems those ribs meddle On a standout amongst them [12].

\section{Recommendation}

All patients should be stabilized and clinically examined before any investigations.

Ultrasound chest should be the first bed side imaging done to critically ill patient or unstable patients with respiratory techniques.

Transverse scan avoiding the ribs shadow should be done to obtain best results.

A correlation between the ultrasound findings and clinical examination and the other laboratory investigations done to find the correct diagnosis.

\section{References}

[1] E .Agricola, C. Arbelot, M. Blaivas, B. Bouhemad, R. Copetti, A. Dean, Ultrasound performs better than radiographs. Thorax; Vol.66 (9), PP.8289,2016.

[2] G. Volpicelli, M .Elbarbary, M. Blaivas, D.A. Lichtenstein, G. Mathis, A.W. Kirkpatrick, International evidence-based recommendations for point-of-care lung ultrasound. Intensive Care Medicine, Vol.38(4), PP.577-91,2014.

[3] A. Mohsen, W. Samy, H. El-Azizy, M. Shehata, Lung ultrasound in intensive care unit: a prospective comparative study with bedside chest radiography using computed tomography of chest as a gold standard. Research and Opinion in Anesthesia and Intensive Care, Vol.5(2), PP.1104,2018 .

[4] N .Xirouchaki, E .Magkanas, K. Vaporidi, E. Kondili, M. Plataki, A. Patrianakos, Lung ultrasound in critically ill patients: comparison with bedside chest radiography. Intensive Care Medicine, Vol.37(9), PP.1488,2017.

[5] B. Bouhemad, H. Brisson, M. Le-Guen, C. Arbelot, Q. Lu, J.J. Rouby, Bedside ultrasound assessment of positive end-expiratory pressure-induced lung recruitment. Am J RespirCrit Care Med, Vol.183(3), PP.341-7,2011.

[6] D.M. Tierney, L.L. Boland, J.D. Overgaard, J.S. Huelster, A. Jorgenson, J.P. Normington, Pulmonary ultrasound scoring system for intubated critically ill patients and its association with clinical metrics and mortality: A prospective cohort study. Journal of Clinical Ultrasound, Vol.46(1), PP.14-22,2018.

[7] S .Mongodi, F. Mojoli, A. Stella, I .Godi, G. Via, G. Tavazzi, Lung ultrasound aeration assessment: comparison of two techniques. Critical Care, Vol.19(1), PP. 222,2015.

[8] A.N. Rubinowitz, M.D. Siegel, I .Tocino, Thoracic imaging in the ICU. Crit Care Clin, Vol.23, PP.539-573,2007.

[9] C.I. Henschke, D.F. Yankelevitz, A. Wand, S.D. Davis, M. Shiau, Accuracy and efficacy of chest 
radiography in the intensive care unit. Radiol Clin North Am, Vol.34, PP.21-31,1996.

[10] S. Mongodi, F. Mojoli, A. Stella1, I .Godi1, G .Via1, G. Tavazzi1, A. Orlando1, B .Bouhemad, Lung ultrasound aeration assessment: comparison of two techniques Critical Care , Vol.19(1),pP222,2015.

[11] I .Ibrahim, Elmahalawy, M. Nagwa, Doha , M.Osama Ebeid , A .Mohammed, Abdel-Hady ,
Ola Saied, Role of thoracic ultrasound in diagnosis of pulmonary and pleural diseases in critically ill patients, Egyptian Journal of Chest Diseases and Tuberculosis , Vol.66, PP. 261-266,2017.

[12] N. Xirouchaki, K. Vaporidi, E. Kondili , M. Plataki, E. Akoumianaki, D. Georgopoulos, Lung ultrasound in critically ill patients: comparison with bedside chest radiography, Intensive Care Med, Vol.37, PP.1488-1493,2011. 